\title{
Analysis of systematic errors of the photoelectric astrolabe catalog SIPA1
}

\author{
Lu Chunlin $^{1,2}, \mathbf{X u}$ Jiayan $^{3}$, Li Dongming ${ }^{1}$, and Liu Jinmei ${ }^{3}$ \\ 1 Purple Mountain Observatory, Nanjing 210008, China \\ e-mail: luchln@public1.ptt.js.cn \\ 2 National Astrononical Observatories, Chinese Academy of Sciences, China \\ 3 Shaanxi Astronomical Observatory, P.O.Box 18, Lintong, Xian 710600, China \\ e-mail: kyc@ms.sxso.ac.cn
}

Received November 12, 1998; accepted May 12, 1999

\begin{abstract}
Systematic errors of the catalog obtained with the observation of Photoelectic Astrolabe Mark I (PHA I) of Shaanxi Astronomical Observatory, mounted at Irkutsk, Russia, are analyzed with reference to the Hipparcos catalog. It is shown that the systematic errors of the astrolabe catalog can be removed effectively using the Hipparcos Catalog as reference and that a star position system with high precision can be obtained.
\end{abstract}

Key words: astrometry — reference system

\section{Instruction}

The Chinese Photoelectric Astrolabe Mark I (PHA I) was improved significantly in 1994. One of the most important changes was to replace the old prism of $30^{\circ}$ zenith distance by a new $45^{\circ}$ prism. The structure and material of the new prism are the same as that of PHA II. It will offer a stable zenith distance of almucantar (Xu et al. 1993). The observation of an astrolabe working at almucantar with zenith distance of $45^{\circ}$ at high latitude (such as Irkutsk, $\varphi=52.2$ ) can give a continuous declination system, besides right ascension system ( $\mathrm{Li}$ et al. 1983; Xu et al. 1998).

The PHA I was moved to the astronomical base of VS NIIFTRI in Irkutsk, Russia $\left(\varphi=52^{\circ} 2\right)$ in Nov. 1995. From Nov. 1995 to June 1997, 200 stars were observed with PHA I. The data were reduced and a catalog named SIPA1 was compiled. SIPA1 covers 65 degrees of declination (from 9.6 to $74^{\circ} 4$ ), which is the first astrolabe catalog without a blind zone in declination (Xu et al. 1998).

$\overline{\text { Send offprint requests to: }}$ Lu Chunlin
The internal errors of SIPA1 have been estimated by $\mathrm{Xu}$ et al. (1998). What is the behavior of its systematic errors? It is important for us to assess the performance of PHA I and its future application. In this paper, the systematic errors of SIPA1 are analysed with the Hipparcos Catalog (ESA, 1997) as reference system. After removing the systematic errors, it is found that the precision of SIPA1 is comparable to the most accurate meridian observations.

\section{Systematic errors of SIPA1}

SIPA1 consists of 200 stars, including 97 FK5 stars and 103 FK5 Extension stars with $9.6<\delta<74$. 4 . To determine the systematic errors of SIPA1, 199 common stars between SIPA1 and Hipparcos catalogs were used. The positions of Hipparcos stars were reduced to the observational epoch of SIPA1 and the difference $\Delta \alpha \cos \delta, \Delta \delta$ were obtained in the sense of SIPA1 - Hipparcos, which are shown in Figs. 1a,b. The systematic differences as a function of right ascension, $\alpha, \Delta \alpha_{\alpha}$ and $\Delta \delta_{\alpha}$, are negligible.

The adopted values of latitude and instrument zenith distance in the reduction of SIPA1 are based on the FK5 system. As is well known, the FK5 catalog, especially the FK5 Extension stars, have zonal systematic difference relative to the Hipparcos catalog. The difference $\Delta \delta$ between the SIPA1 input catalog, which consists of FK5 and FK5 Extension stars, and the Hipparcos catalog at the epoch of SIPA1 observations are shown in Fig. 2. The systematic errors of FK5 may be introduced into SIPA1 in the following form (Li et al. 1983):

$\Delta \delta=\Delta \varphi \sec z \sec \varphi \cos \delta-(\Delta z+\Delta \varphi \tan \varphi \tan z) / \cos q(1)$ where $q$ is the parallactic angle, $\varphi$ is the latitude and $z$ is the zenith distance. With a least squares fit to the $\Delta \delta$ 

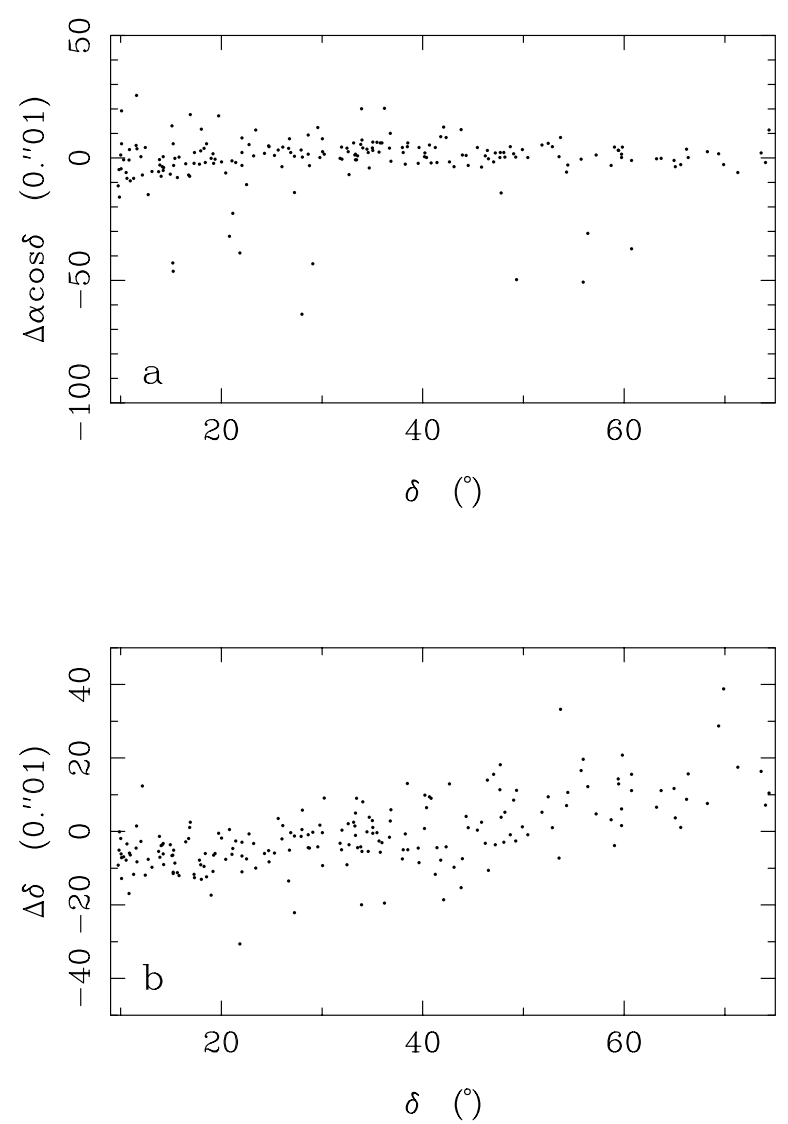

Fig. 1. a-b) The difference between SIPA1 and the Hipparcos catalog. a) $\Delta \alpha \cos \delta$ vs. $\delta$. b) $\Delta \delta$ vs. $\delta$

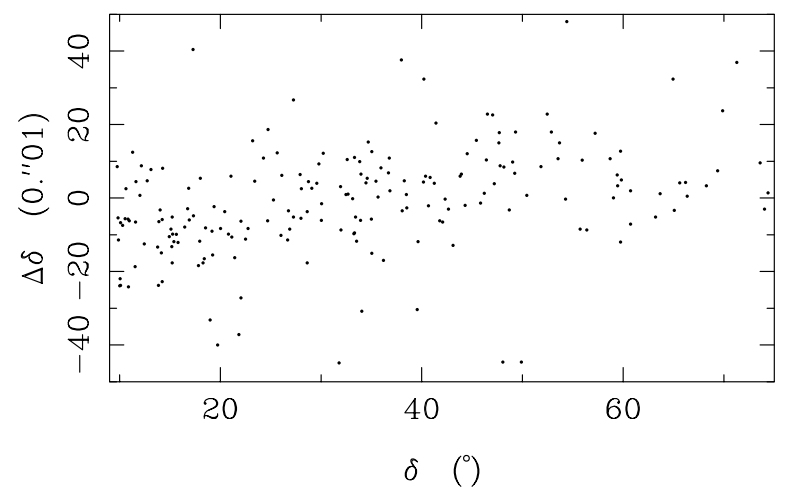

Fig. 2. The difference $\Delta \delta$ between the SIPA1 Input catalog and the Hipparcos catalog at the epoch of SIPA1 observations

Table 1. Comparison of the precision of SIPA1 and Meridian catalogs

\begin{tabular}{lcc}
\hline Catalog & $\Delta \alpha \cos \delta$ & $\Delta \delta$ \\
\hline CAMC7 & $\pm 0 . .067$ & $\pm 0^{\prime \prime} 087$ \\
Bordeaux & $\pm 0^{\prime \prime} 045$ & $\pm 0 . .068$ \\
Tokyo89 & $\pm 0^{\prime \prime} .135$ & $\pm 0^{\prime \prime} .135$ \\
SIPA1 & $\pm 0^{\prime \prime} 070$ & $\pm 0 . .072$ \\
\hline
\end{tabular}

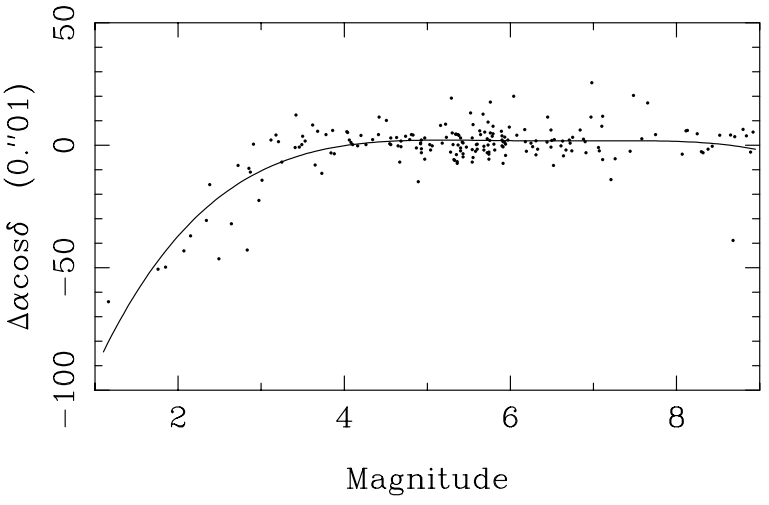

Fig. 3. Magnitude equation of SIPA1, in which the solid line is calculated from Eq. (3)

values of Fig. 1b, the systematic differences in 0. . 01 in declination between SIPA1 and Hipparcos are derived as:

$\Delta \delta=0.464 / \cos q+35.40 \cos \delta-28.0$.

It can be seen from Fig. 3 that there exists a significant magnitude equation in the right ascension system of SIPA1, which can be expressed with a forth order polynomial:

$$
\begin{array}{r}
\Delta \alpha \cos \delta=187.8-122.72 V+29.399 V^{2} \\
-3.0939 V^{3}+0.12084 V^{4}
\end{array}
$$

where $V$ is the visual magnitude of stars. The unit in Eq. (3) is in $0 . \prime 01$.

\section{Precision estimation of SIPA1 and comparison with meridian catalogs}

The rms differences of SIPA1 and Hipparcos catalogs are \pm 0 '. 118 and \pm 0. . 095 for $\Delta \alpha \cos \delta$ and $\Delta \delta$ respectively. It can be seen from Fig. 1a and Fig. 3 that the large rms difference for $\Delta \alpha \cos \delta$ is mainly caused by those stars brighter than 3th magnitude, and that the right ascension system of SIPA1 coincides with the Hipparcos system very well, except for those bright stars. The amplitude of systematic errors in declination, $\Delta \delta_{\delta}$, are about -0.03 to +0 .'03. Removing the systematic differences from SIPA1 using Eq. (2) and Eq. (3), the remain differences are shown in Figs. 4a,b. And the rms differences are reduced to $\pm 00^{\prime \prime} 070$ and \pm 0 . 072 for $\Delta \alpha \cos \delta$ and $\Delta \delta$ respectively.

To judge the level of Photoelectric Astrolabe catalog, we compared SIPA1 with the most productive automatic meridian circles, Carlsberg, Bordeaux and Tokyo. First, the CAMC7 (CAMC Consortium 1992) and TMC89 (Yoshizawa et al. 1993) are compared with the Hipparcos catalog respectively. After removing systematic differences, the rms differences of $\Delta \alpha \cos \delta$ and $\Delta \delta$ are calculated. For the Bordeaux meridian circle, the comparison 

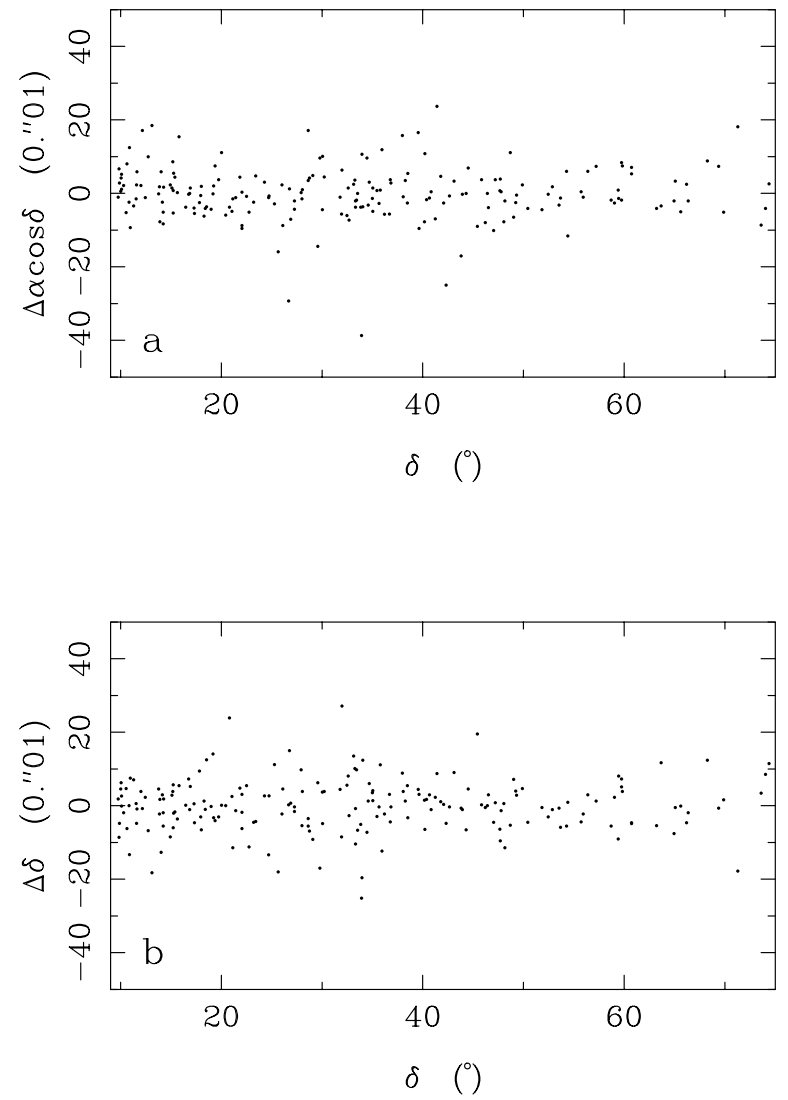

Fig. 4. a,b) The difference between SIPA1 and Hipparcos cata$\log$ after removing the systematic errors. a) $\Delta \alpha \cos \delta$ vs. $\delta$. b) $\Delta \delta$ vs. $\delta$

between Hipparcos and 173 radio stars observed with the Bordeaux meridian circle is used (Requième et al. 1995). The results are given in Table 1.

\section{Conclusion}

The precision of the declination system of SIPA1 cata$\log$ is as high as the best modern meridian circles, the right ascension system is degraded a little by a magnitude equation. If the problem of the magnitude equation in the photoelectric recording device can be resolved, the precision of right ascension observation will be improved significantly. The PHA I still can play important role in the maintenance of the Hipparcos proper motion system and in the improvement of the link between the Hipparcos and radio reference systems by long-term observation of radio stars.

Acknowledgements. The authors would like to give many thanks to the China National Natural Science Foundation, to the Bureau of Basic Research and Bureau of International Cooperation of the Chinese Academy of Sciences, and the China Astronomical Committee, which give great support to our research.

\section{References}

CAMC Consortium, 1992, Carlsberg Meridian Catalog, La Palma, No. 7

ESA, 1997, The Hipparcos and Tycho Catalogues, ESA SP-1200

Li Dongming, Xu Jiayan, Luo Dingjiang, 1983, Method of Equal Altitude And Its Application In Fundamental Astrometry. Science Press, Beijing

Réquième Y., et al., A\&A 304, 121

Xu Jiayan, et al., 1993, A\&A 271, 360

Xu Jiayan, et al., 1998, A\&AS 132, 233

Yoshizawa M., et al., 1993, Publ. Nat. Astron. Obs. Jpn 3, 45 